Calcium glomerular filtration

rate

kidney parathyroid glands phosphate reabsorption rickets vitamin D

\title{
The Effect of Vitamin D on Renal Inorganic Phosphate Reabsorption of Normal Rats, Parathyroidectomized Rats, and Rats with Rickets
}

\author{
D. Gekle [46], J. Ströder, and D. Rostock \\ Kinderklinik der Universität Würzburg, Würzburg, Germany
}

Extract

In order to determine the effect of vitamin $D$ on tubular reabsorption of phosphate, micropuncture studies were performed in the proximal convolution of normal rats, of parathyroidectomized rats, and of rats with rickets. The studies showed that vitamin $\mathrm{D}$ exerted a direct effect on transtubular reabsorption of phosphate that was independent of parathyroid function. The glomerular filtration rate was not affected by vitamin $\mathrm{D}$. The reabsorption of phosphate of normal rats was $13.51 \mu \mathrm{moles} / \mathrm{min}$ per $\mathrm{kg}$. This corresponds to a fractional reabsorption of $73 \%$. With vitamin $\mathrm{D}$ the fractional reabsorption increased to $86 \%$. Despite continuous infusion of vitamin $\mathrm{D}$, the phosphate reabsorption decreased to $13.95 \mu \mathrm{moles} / \mathrm{min} / \mathrm{kg}$ (fractional reabsorption 74\%) after 180-240 min. Parathyroidectomized rats also showed a decreased transtubular reabsorption of phosphate during vitamin $\mathrm{D}$ infusion. In animals under a phosphate load who suffered from rickets, it was possible to normalize the decreased rate of reabsorption of phosphate (fractional reabsorption $59 \%$ ) by the administration of vitamin D (fractional reabsorption $78 \%$ ).

\section{Speculation}

We were unable to determine why, despite continuous infusion of vitamin $D$ and independent of the dosage, a decrease occurred in the increased reabsorption after 90-120 min and why after 180-240 min this function returned to the starting value. Since at this latter time toxic renal damage is unlikely and renal function was controlled during the whole experiment, the question arises whether inadequate activation, reactive breakdown of vitamin $\mathrm{D}$, or development of resistance to the physiological activity of the vitamin is involved.

\section{Introduction}

Despite numerous studies regarding the mode of action of vitamin D, its action on renal transport of phosphate is still obscure. A few authors postulated, primarily in cases of vitamin D-deficient rickets, that increased reabsorption of phosphate occurs $[13,14,19$,
$26,28,29]$. Other authors, in cases with hypoparathyroidism and during administration of high doses of vitamin $\mathrm{D}$, reported an inhibition of tubular reabsorption of phosphate $[1,5,23]$. It is not clear whether vitamin $D$ directly affects tubular reabsorption of vitamin $\mathrm{D}[13,14]$, or whether the changed reabsorption 
is the result of the action of vitamin $\mathrm{D}$ on the parathyroid glands $[19,23]$. Ney et al. [23] have reiterated the opinion that vitamin $\mathrm{D}$ inhibits the function of the parathyroid glands and through this mechanism produces an increase in the rate of reabsorption of phosphate by the kidney. Au et al. [3] and Ham et al. [12] reported that the function of the parathryoid glands relates exclusively to the calcium level in serum as a feedback mechanism. These findings were countered by studies $[7,19,26,33]$ of healthy organisms, humans and animals, which show that vitamin $D$ has no effect on clearance of phosphate. Since disagreement between authors can partly be explained by the method and partly by the heterogenous test material, we performed micropuncture studies at the proximal convolution of the kidney of normal rats, of parathyroidectomized rats, and of rats with rickets.

Results show that vitamin D increased tubular reabsorption of phosphate in normal rats as well as in parathyroidectomized rats and in rats with rickets. With continuous infusion of vitamin $\mathrm{D}$ the increased reabsorption again decreased after $3-4 \mathrm{hr}$. The reabsorption mechanism in the epithelium of the tubule appears independent of the function of the parathyroid glands.

\section{Materials and Methods}

Male rats of the Wistar strain, weighing 180-200 g [36], received a standard diet [37] with a calcium-tophosphorus ratio of $1: 112 \mathrm{hr}$ prior to the test; after that, only water was provided ad libitum.

\section{Micropuncture}

The animals were anesthetized by intraperitoneal injection of inactin $(80-100 \mathrm{mg} / \mathrm{kg})$, and positioned on a heated operating table $\left(37^{\circ}\right)$. The body temperature of the rats was continuously monitored and controlled. After tracheotomy and cannulation of the left and right jugular vein the left kidney was exposed by a loin incision, and immobilized in situ in a Plexiglas clish. Without injuring the capsule, fat and connective tissue were carefully dissected from the surface of the kidney. During the test the kidney was covered with paraffin oil at $37^{\circ}$. A lamp [38] was used to illuminate the surface of the kidney and a stereomicroscope [38] (100fold magnification) was used for examination. All test animals received an infusion of $0.86 \% \mathrm{NaCl}$ via the right jugular vein. The infusion rate was $1-1.5 \mathrm{ml} / \mathrm{hr}$. Depending on the problem under examination this intravenous solution contained $1.800-20$ units of vi$\operatorname{tamin} \mathrm{D}_{3}$ [39] per min per $100 \mathrm{~g}$ body wt. Blood sam- ples were drawn from a tail vein which had been made hyperemic. A polyethylene catheter was tied into the ureter slightly distal to the renal pelvis for continuous urine collection. Micromanipulators [38] were used for micropuncture. Removal of tubular fluid from the proximal convolution under free-flow conditions was carried out by using oil-filled micropuncture capillaries with an external diameter of the tip of 8-10 $\mu$. At the end of test the punctured tubules were filled with latex and were measured by microdissection. The distance from the point of puncture to the glomerulus was reported as a percentage of the total length of the proximal tubule.

Glomerular filtration rate was measured as clearance of ${ }^{14} \mathrm{C}$-inulin and ${ }^{51} \mathrm{Cr}$ ethylenediaminetetraacetate (EDTA) [40] after it was found that the latter is identical with clearance of ${ }^{14} \mathrm{C}$-inulin [9]. The animals received an initial dose either of $100 \mu \mathrm{Ci}{ }^{51} \mathrm{Cr}$-EDTA or ${ }^{14} \mathrm{C}$-inulin; subsequent to this, they received $100 \mu \mathrm{Ci}$

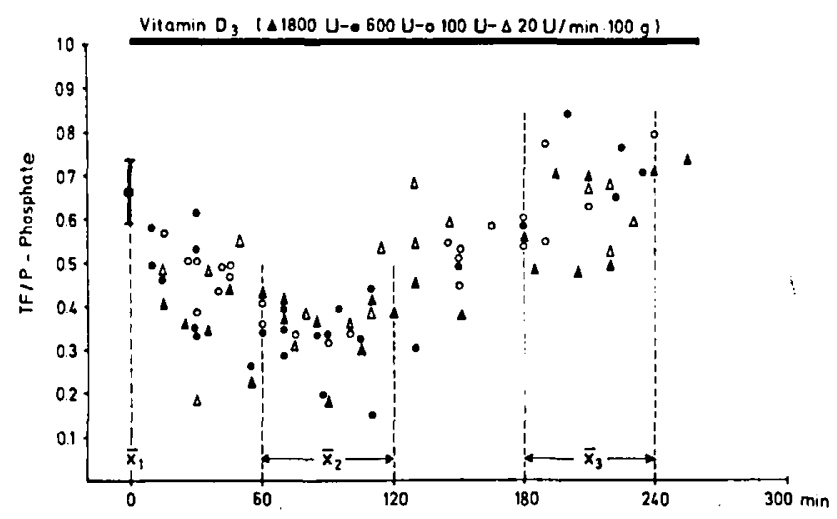

Fig. 1. The effect of various doses of vitamin $\mathrm{D}$ on the TF/P-phosphate of normal animals continuously infused with vitamin $D$. Vitamin $D_{s}$ dose: $\Delta$ 1,800 U; $\bullet 600 \mathrm{U} ; \bigcirc 100 \mathrm{U} ; \Delta 20 \mathrm{U} / \mathrm{min} / 100$ g.
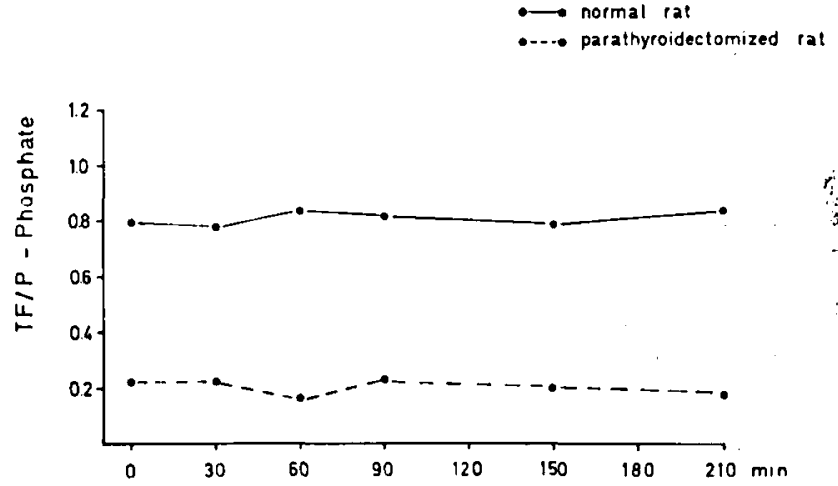

Fig. 2. The effect of infusion of $0.86 \% \mathrm{NaCl}(1.5 \mathrm{ml} / \mathrm{hr})$ on the TF/P-phosphate in a normal and parathyroidectomized rat. 
Table $I$. Control animals

\begin{tabular}{|c|c|c|c|c|c|c|c|c|}
\hline Rat & $\begin{array}{c}\text { Plasma } \\
\text { phosphate } \\
\text { mmoles }\end{array}$ & $\begin{array}{l}\text { Tubular } \\
\text { phosphate } \\
\text { er }\end{array}$ & TF/P-phosphate & $\begin{array}{c}\text { Tubule } \\
\text { length, } \\
\%\end{array}$ & $\begin{array}{l}\mathrm{TF} / \mathrm{P}- \\
\text { inulin }\end{array}$ & $\begin{array}{c}\text { Tubule } \\
\text { length, } \\
\%\end{array}$ & $\mathrm{GFR}, \mathrm{ml} / \mathrm{min} / \mathrm{kg}$ & $\begin{array}{l}\text { Serum calcium, } \\
\mathrm{mg} / 100 \mathrm{ml}\end{array}$ \\
\hline \multirow[t]{4}{*}{$\mathbf{I}$} & 2.41 & 1.97 & 0.82 & 28 & 1.96 & 40 & 8.50 & 10.2 \\
\hline & & 1.68 & 0.70 & 30 & 1.70 & 30 & 8.70 & \\
\hline & & 1.97 & 0.82 & 23 & & & 8.70 & \\
\hline & & & & & & & 9.18 & \\
\hline \multirow[t]{3}{*}{ II } & 4.00 & 2.41 & 0.60 & 50 & 1.42 & 30 & 6.18 & 10.0 \\
\hline & & 2.72 & 0.68 & 34 & 1.60 & 30 & 8.94 & \\
\hline & & 2.60 & 0.65 & 30 & 1.70 & 25 & 8.60 & \\
\hline \multirow[t]{3}{*}{ III } & 3.60 & 2.45 & 0.68 & 48 & 1.30 & 25 & 5.40 & 9.9 \\
\hline & & 2.16 & 0.60 & 50 & 1.60 & 30 & 4.70 & \\
\hline & 3.58 & 2.22 & 0.62 & 60 & 2.09 & 46 & & \\
\hline \multirow[t]{7}{*}{ IV } & 2.71 & 1.90 & 0.70 & 44 & 2.50 & 50 & 4.70 & 10.2 \\
\hline & 2.68 & 1.80 & 0.67 & 47 & 1.70 & 40 & 5.80 & \\
\hline & 2.66 & 1.60 & 0.60 & 54 & 2.25 & 38 & 5.90 & \\
\hline & 2.69 & 2.10 & 0.78 & 26 & & & 5.80 & \\
\hline & 2.81 & 2.19 & 0.78 & 25 & & & & \\
\hline & & 1.96 & 0.70 & 15 & & & & \\
\hline & & 2.08 & 0.74 & 35 & & & & \\
\hline \multirow[t]{4}{*}{ V } & 2.40 & 1.44 & 0.60 & 40 & 2.90 & 63 & 8.70 & 10.3 \\
\hline & 2.40 & 1.65 & 0.69 & 35 & 3.00 & 60 & 8.22 & \\
\hline & 2.42 & 1.33 & 0.55 & 30 & & & 8.40 & \\
\hline & 2.42 & 1.28 & 0.53 & 35 & & & & \\
\hline \multirow[t]{2}{*}{$\mathrm{VI}$} & 2.36 & 1.67 & 0.71 & 40 & 2.30 & 57 & 5.00 & 9.8 \\
\hline & & & & & & & 5.26 & \\
\hline \multirow[t]{3}{*}{ VII } & 2.43 & 1.38 & 0.57 & 38 & 2.70 & 52 & & 11.0 \\
\hline & & 1.82 & 0.75 & 26 & & & & \\
\hline & & 1.73 & 0.71 & 26 & & & & \\
\hline \multirow[t]{3}{*}{ VIII } & 3.48 & 1.98 & 0.57 & 27 & 2.90 & 52 & 6.36 & 10.4 \\
\hline & & 2.26 & 0.65 & 41 & & & 7.76 & \\
\hline & & 2.33 & 0.67 & 33 & & & & \\
\hline \multirow[t]{3}{*}{ IX } & 2.80 & 1.73 & 0.62 & 44 & 2.80 & 46 & 5.10 & 10.0 \\
\hline & & 1.62 & 0.58 & 39 & & & 6.66 & \\
\hline & 2.61 & 1.41 & 0.54 & 55 & 2.50 & 43 & 5.32 & \\
\hline \multirow[t]{4}{*}{$\mathrm{x}$} & 2.38 & 1.59 & 0.66 & 20 & & & & 9.8 \\
\hline & 2.12 & 1.50 & 0.71 & 50 & 2.20 & 45 & & \\
\hline & 2.12 & 1.59 & 0.75 & 35 & & & & \\
\hline & 2.20 & 1.85 & 0.84 & 20 & & & & \\
\hline Mean $\pm s D$ & $2.69 \pm 0.52$ & & $0.67 \pm 0.09$ & & & & $6.86 \pm 1.62$ & $10.16 \pm 0.36$ \\
\hline
\end{tabular}

${ }^{51} \mathrm{Cr}$-EDTA or ${ }^{14} \mathrm{C}$-inulin per hr intravenously via the jugular vein. Radioactivity was measured with a liquid scintillation counter [4l]. Ratios of TF/P-inulin were determined with ${ }^{14} \mathrm{C}$-inulin $(\mathrm{TF}=$ concentration of inulin in tubular fluid; $\mathbf{P}=$ concentration of inulin in plasma).

\section{Determination of Inorganic Phosphate}

Before the plasma was used, proteins were removed with $5 \%$ trichloroacetic acid. The concentration of inorganic phosphate in the tubular fluid and in the plasma was determined with the aid of an ultramicromethod which had been developed by modifying the 
Table II. Normal rats infused with vitamin $D_{3}$

\begin{tabular}{|c|c|c|c|c|c|c|c|c|}
\hline Rat & $\begin{array}{c}\ell^{b} \\
\text { min after } \\
\text { infusion }\end{array}$ & $\begin{array}{c}\text { Plasma } \\
\text { phosphate } \\
\text { mmoles }\end{array}$ & $\begin{array}{l}\text { Tubular } \\
\text { phosphate } \\
\text { liter }\end{array}$ & $\begin{array}{c}\text { TF/P- } \\
\text { phosphate }\end{array}$ & $\begin{array}{c}\text { Tubule } \\
\text { length, } \\
\%\end{array}$ & TF/P-inulin & GFR, $\mathrm{ml} / \mathrm{min} / \mathrm{kg}$ & $\begin{array}{c}\text { Serum } \\
\text { calcium, } \\
\mathrm{mg} / 100 \mathrm{ml}\end{array}$ \\
\hline \multirow[t]{8}{*}{$I, 1800^{3}$} & 15 & 2.43 & 1.00 & 0.41 & 20 & & & \\
\hline & 35 & 2.43 & 0.85 & 0.35 & 46 & & & \\
\hline & 70 & 2.43 & 1.00 & 0.41 & 25 & & 5.76 & 9.2 \\
\hline & 85 & 3.10 & 1.05 & 0.34 & 40 & & 7.66 & \\
\hline & 120 & 3.10 & 1.21 & 0.39 & 45 & & 5.86 & \\
\hline & 150 & 3.10 & 1.18 & 0.38 & 50 & 2.44 & & \\
\hline & 195 & 2.51 & 1.78 & 0.71 & 38 & & 6.06 & \\
\hline & 210 & 2.51 & 1.76 & 0.70 & 42 & & 9.40 & 9.3 \\
\hline \multirow[t]{8}{*}{ II, $1800^{1}$} & 0 & 3.19 & 1.82 & 0.69 & 52 & & & \\
\hline & 55 & 3.19 & 0.73 & 0.23 & 56 & 2.30 & & \\
\hline & 70 & 3.19 & 1.21 & 0.38 & 27 & & 7.58 & \\
\hline & 90 & 3.19 & 0.60 & 0.19 & 61 & 2.38 & 6.66 & 8.6 \\
\hline & 105 & 3.22 & 1.00 & 0.31 & 39 & & 8.50 & \\
\hline & 185 & 3.22 & 1.54 & 0.48 & 19 & & 7.12 & \\
\hline & 205 & 3.22 & 1.51 & 0.47 & 44 & & 5.90 & \\
\hline & 225 & 3.12 & 1.53 & 0.49 & 31 & & 7.20 & 8.6 \\
\hline \multirow[t]{9}{*}{ III, $1800^{1}$} & 0 & 2.40 & 1.60 & 0.67 & 59 & & & \\
\hline & 25 & 2.40 & 0.89 & 0.37 & 63 & 1.94 & & \\
\hline & 45 & 2.40 & 1.08 & 0.45 & 58 & 2.02 & & \\
\hline & 60 & 2.40 & 1.03 & 0.43 & 42 & & 7.70 & 9.4 \\
\hline & 110 & 2.44 & 1.05 & 0.43 & 57 & & 7.40 & \\
\hline & 130 & 2.44 & 1.12 & 0.46 & 63 & 1.68 & & \\
\hline & 180 & 2.44 & 1.36 & 0.56 & 24 & 1.16 & 5.02 & \\
\hline & 240 & 2.38 & 1.69 & 0.71 & 59 & 2.61 & 7.00 & 11.4 \\
\hline & 255 & 2.38 & 1.76 & 0.74 & 45 & & 8.26 & \\
\hline \multirow[t]{5}{*}{$\mathrm{IV}, 600^{\mathrm{I}}$} & 10 & 2.97 & 1.42 & 0.48 & 64 & & & \\
\hline & 15 & 2.97 & 1.39 & 0.47 & 53 & & & \\
\hline & 55 & 3.06 & 0.82 & 0.27 & - & & & \\
\hline & 70 & 3.06 & 0.88 & 0.29 & 40 & 2.28 & 5.52 & 9.8 \\
\hline & 130 & 3.06 & 0.95 & 0.31 & 37 & & & \\
\hline \multirow[t]{6}{*}{$\mathrm{V}, 600^{1}$} & 0 & 2.20 & 1.63 & 0.74 & 62 & & & \\
\hline & 35 & 2.20 & 0.77 & 0.35 & 43 & & & \\
\hline & 70 & 2.20 & 0.77 & 0.35 & - & & 5.74 & 9.8 \\
\hline & 85 & 2.45 & 0.90 & 0.37 & 50 & & 6.76 & \\
\hline & 200 & 2.49 & 2.11 & 0.85 & 61 & 2.45 & 7.52 & \\
\hline & 225 & 2.49 & 1.92 & 0.77 & 55 & 2.51 & 8.08 & 9.6 \\
\hline \multirow[t]{4}{*}{ IV, $600^{1}$} & 0 & 2.61 & 1.73 & 0.66 & 54 & & & \\
\hline & 70 & 2.97 & 1.10 & 0.37 & 50 & & & \\
\hline & 95 & 2.42 & 0.96 & 0.40 & - & & & \\
\hline & 105 & 2.42 & 0.80 & 0.33 & - & & & \\
\hline \multirow[t]{6}{*}{ VII, $600^{1}$} & 0 & 2.00 & 1.25 & 0.62 & 40 & & & \\
\hline & 10 & 2.00 & 1.18 & 0.59 & 43 & & & \\
\hline & 30 & 2.00 & 1.08 & 0.54 & 60 & & & \\
\hline & 60 & 2.00 & 0.70 & 0.35 & 26 & & 7.24 & \\
\hline & 90 & 2.00 & 0.36 & 0.18 & - & & & 9.4 \\
\hline & 110 & 2.10 & 0.31 & 0.15 & 44 & & & \\
\hline Mean \pm SD & $\begin{array}{l}\bar{X}_{2} \\
\bar{X}_{2}\end{array}$ & & & & & & $\begin{array}{l}6.86 \pm 0.96 \\
7.16 \pm 1.27\end{array}$ & \\
\hline
\end{tabular}


Table II-Continued

\begin{tabular}{|c|c|c|c|c|c|c|}
\hline Rat & $\underset{\substack{\min \text { after } \\
\text { infusion }}}{\stackrel{,}{y^{2}}}$ & $\begin{array}{l}\text { Plasma phosphate } \\
\text { mmoles/liter }\end{array}$ & $\begin{array}{l}\text { Tubular } \\
\text { phosphate }\end{array}$ & TF/P-phosphate & $\begin{array}{c}\text { Tubule length, } \\
\%\end{array}$ & $\begin{array}{l}\text { Serum calcium, } \\
\mathrm{mg} / 100 \mathrm{ml}\end{array}$ \\
\hline \multirow[t]{9}{*}{ VIII, $600^{1}$} & 0 & 2.36 & 1.84 & 0.78 & 45 & \\
\hline & 30 & 2.36 & 1.46 & 0.62 & 40 & \\
\hline & 70 & 2.36 & 0.94 & 0.40 & - & \\
\hline & $\cdot 90$ & 2.36 & 0.78 & 0.33 & - & 9.6 \\
\hline & 110 & 2.36 & 1.04 & 0.44 & - & \\
\hline & 150 & 2.15 & 1.09 & 0.51 & 45 & \\
\hline & 180 & 2.15 & 1.25 & 0.58 & 25 & 10.4 \\
\hline & 225 & 2.15 & 1.39 & 0.65 & 30 & \\
\hline & 240 & 2.15 & 1.52 & 0.71 & - & \\
\hline \multirow[t]{7}{*}{ IX, $100^{2}$} & 0 & 2.27 & 1.61 & 0.71 & - & \\
\hline & 15 & 2.27 & 1.29 & 0.57 & 54 & \\
\hline & 30 & 2.27 & 1.16 & 0.51 & 61 & \\
\hline & 45 & 2.27 & 1.13 & 0.50 & 30 & \\
\hline & 60 & 2.29 & 0.82 & 0.36 & 35 & \\
\hline & 145 & 2.29 & 1.26 & 0.55 & 33 & \\
\hline & 190 & 2.26 & 1.76 & 0.78 & 40 & \\
\hline \multirow{9}{*}{$\mathrm{X}, 100^{1}$} & 30 & 2.32 & 1.18 & 0.51 & 45 & \\
\hline & 45 & 2.32 & 1.18 & 0.51 & - & \\
\hline & 60 & 2.32 & 0.95 & 0.41 & 31 & \\
\hline & 75 & 2.32 & 0.78 & 0.34 & 39 & 9.8 \\
\hline & 90 & 2.32 & 0.76 & 0.33 & 26 & \\
\hline & 150 & 2.38 & 1.07 & 0.45 & 25 & \\
\hline & 165 & 2.38 & 1.40 & 0.59 & 50 & \\
\hline & 180 & 2.38 & 1.43 & 0.60 & 24 & \\
\hline & 210 & 2.38 & 1.50 & 0.63 & - & 10.0 \\
\hline \multirow[t]{9}{*}{$\mathrm{XI}, 100^{1}$} & 0 & 3.04 & 2.10 & 0.70 & - & \\
\hline & 30 & 3.04 & 1.18 & 0.39 & 42 & \\
\hline & 40 & 3.04 & 1.34 & 0.44 & 45 & \\
\hline & 50 & 3.04 & 1.40 & 0.46 & - & \\
\hline & 100 & 3.14 & 1.06 & 0.34 & - & \\
\hline & 150 & 3.14 & 1.66 & 0.53 & 50 & \\
\hline & 180 & 3.14 & 1.73 & 0.55 & 48 & \\
\hline & 190 & 3.14 & 1.69 & 0.54 & 62 & \\
\hline & 240 & 3.14 & 2.51 & 0.80 & - & \\
\hline \multirow[t]{8}{*}{ XII, $20^{1}$} & 15 & 2.34 & 1.12 & 0.48 & - & \\
\hline & 30 & 2.34 & 0.44 & 0.19 & - & \\
\hline & 80 & 2.34 & $0.9 \mathrm{l}$ & 0.39 & 一 & \\
\hline & 110 & 2.34 & 0.93 & 0.40 & - & \\
\hline & 130 & 2.30 & 1.26 & 0.55 & - & \\
\hline & 145 & 2.30 & 1.38 & 0.60 & - & \\
\hline & 210 & 2.40 & 1.63 & 0.68 & - & 9.3 \\
\hline & 220 & 2.40 & 1.65 & 0.69 & - & \\
\hline \multirow[t]{9}{*}{ XIII, $20^{1}$} & 0 & 2.87 & 1.98 & 0.69 & - & \\
\hline & 35 & 2.87 & 1.40 & 0.49 & - & \\
\hline & 50 & 2.87 & 1.61 & 0.56 & - & \\
\hline & 75 & 2.87 & 0.97 & 0.34 & 一 & \\
\hline & 100 & 2.87 & 1.03 & 0.36 & 42 & 9.6 \\
\hline & 115 & 2.87 & 1.55 & 0.54 & - & \\
\hline & 130 & 2.76 & 1.93 & 0.70 & - & \\
\hline & 220 & 2.76 & 1.41 & 0.51 & 62 & \\
\hline & 230 & 2.76 & 1.63 & 0.59 & - & \\
\hline \multirow[t]{2}{*}{ Mean \pm sD } & $\overline{X_{2}}$ & $2.59 \pm 0.40$ & - & $0.35 \pm 0.11$ & - & $9.47 \pm 0.34$ \\
\hline & $X_{3}$ & $2.62 \pm 0.37$ & - & $0.64 \pm 0.11$ & - & $9.80 \pm 0.91$ \\
\hline
\end{tabular}

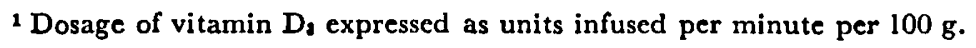


method of Lowry et al. [22]. The principle of the reaction is as follows: phosphoric acid and ammonium molybdate change to ammonium phosphate molyb. date, and it is then reduced by ascorbic acid to molybdate-blue.

\section{Analytic Method}

The sample, $20 \times 10^{-6} \mathrm{ml}$, which had been stored under paraffin oil, was mixed in a wet chamber on Parafilm with $1 \times 10^{-2} \mathrm{ml}$ of a test solution $(1 \mathrm{ml} 10 \mathrm{~N}$ $\mathrm{H}_{2} \mathrm{SO}_{4}, 6 \mathrm{ml} \mathrm{H}_{2} \mathrm{O}, 1 \mathrm{ml} 1.5 \%$ ammonium molybdate solution, and $1 \mathrm{ml} 10 \%$ ascorbic acid solution). The sample was then pipetted into a reaction container and stored for $2 \mathrm{hr}$ at $38^{\circ}$ in an incubator. The optical density was determined at $820 \mathrm{~m} \mu$ in a spectrophotometer [42], with the aid of a microcuvette [32]. Ten replications of a $2.5 \mathrm{~mm}$ phosphate solution (volume $20 \times 10^{-6} \mathrm{ml}$ ) were measured with a standard deviation of $\pm 1 \%$.

\section{Parathyroidectomy}

Under light ether anesthesia rats of the Wistar strain, weighing $180-200 \mathrm{~g}$, underwent bilateral parathyroidectomy by electrocoagulation. To check the success of surgery, the calcium level in serum was determined with a flame photometer before parathyroidectomy and then $48-72 \mathrm{hr}$ after parathyroidectomy. The animals were used for the experiments on the 3rd post-operative day when the effect of endogenous parathyroid hormone had completely subsided. At this time the calcium level reached its lowest value.

Table $I I I A$. Parathyroidectomized rats without vitamin $\mathrm{D}_{3}$ infusion

\begin{tabular}{|c|c|c|c|c|c|c|c|}
\hline Rat & $\begin{array}{l}\text { Plasma phosphhate } \\
\text { mmoles/liter }\end{array}$ & $\begin{array}{l}\text { Tubular } \\
\text { phosphate }\end{array}$ & TF/P-phosphate & $\begin{array}{c}\text { Tubule } \\
\text { length, \% }\end{array}$ & TF/P-inulin & $\mathrm{GFR}, \mathrm{ml} / \mathrm{min} / \mathrm{kg}$ & $\begin{array}{l}\text { Serum calcium, mg/100 } \\
\text { ml before/after para- } \\
\text { thyroidectomy }\end{array}$ \\
\hline \multirow[t]{3}{*}{ I } & 3.28 & 0.39 & 0.12 & 42 & 2.01 & 5.72 & $9.9 / 8.2$ \\
\hline & 3.28 & 0.43 & 0.13 & 43 & 2.25 & 5.34 & \\
\hline & 3.28 & 0.52 & 0.16 & 40 & 2.10 & & \\
\hline \multirow[t]{5}{*}{ II } & 3.70 & 0.58 & 0.13 & 47 & & & \\
\hline & 3.63 & 0.40 & 0.11 & 40 & 1.83 & 5.62 & \\
\hline & 3.65 & 0.73 & 0.20 & 40 & 2.04 & 5.14 & $10.0 / 8.0$ \\
\hline & 3.72 & 0.82 & 0.22 & 35 & 1.49 & & \\
\hline & 3.75 & 0.75 & 0.20 & 34 & & & \\
\hline \multirow[t]{3}{*}{ III } & 3.16 & 0.38 & 0.12 & 40 & & & $9.5 / 8.4$ \\
\hline & 3.12 & 0.75 & 0.24 & 33 & 1.98 & 4.56 & \\
\hline & 3.06 & 0.46 & 0.15 & 45 & 1.34 & 4.30 & \\
\hline \multirow[t]{3}{*}{ IV } & 3.10 & 0.68 & 0.22 & 45 & 1.93 & 4.08 & $11.0 / 6.0$ \\
\hline & 3.08 & 0.74 & 0.24 & 26 & & 4.48 & \\
\hline & 3.12 & 0.75 & 0.24 & 22 & 1.77 & 4.56 & \\
\hline \multirow[t]{3}{*}{ V } & 3.66 & 0.84 & 0.23 & 27 & & & $10.1 / 6.8$ \\
\hline & 3.66 & 0.48 & 0.13 & 54 & 2.19 & 5.38 & \\
\hline & 3.66 & 1.28 & 0.35 & 19 & 2.06 & 6.26 & \\
\hline \multirow[t]{4}{*}{ VI } & 3.70 & 0.85 & 0.23 & 36 & & & $10.4 / 6.4$ \\
\hline & 3.71 & 1.48 & 0.40 & 21 & 2.12 & 4.92 & \\
\hline & 3.74 & 0.90 & 0.24 & 39 & 2.07 & 4.96 & \\
\hline & 3.72 & 1.19 & 0.32 & 24 & 2.06 & & \\
\hline \multirow[t]{3}{*}{ VII } & 3.61 & 1.44 & 0.40 & 20 & & & $10.3 / 7.8$ \\
\hline & 3.50 & 0.53 & 0.15 & 36 & 2.07 & & \\
\hline & 3.70 & 0.37 & 0.10 & 50 & 2.06 & & \\
\hline \multirow[t]{4}{*}{ VIII } & 3.20 & 0.67 & 0.21 & 25 & & & $10.6 / 7.2$ \\
\hline & 3.16 & 0.44 & 0.14 & 47 & & & \\
\hline & 3.25 & 0.71 & 0.22 & 30 & & & \\
\hline & 3.09 & 0.46 & 0.15 & 34 & & & \\
\hline Mean \pm sD & $3.40 \pm 0.26$ & & $0.20 \pm 0.08$ & & & $0.53 \pm 0.49$ & \\
\hline
\end{tabular}


Table III B. Parathyroidectomized rats infused with vitamin $\mathrm{D}_{2}(500 \mathrm{U} / \mathrm{min})$

\begin{tabular}{|c|c|c|c|c|}
\hline Rat & $\begin{array}{c}\text { t, min } \\
\text { after } \\
\text { infusion }\end{array}$ & $\begin{array}{l}\text { Plasma } \\
\text { phosphate } \\
\text { mmoles/lit }\end{array}$ & $\begin{array}{l}\text { Tubular } \\
\text { phosphate } \\
\text { ter }\end{array}$ & $\begin{array}{c}\text { TF/P. } \\
\text { phosphate }\end{array}$ \\
\hline \multirow[t]{7}{*}{ IX } & 0 & 3.06 & 0.64 & 0.21 \\
\hline & 40 & 3.06 & 0.43 & 0.14 \\
\hline & 100 & 3.32 & 0.63 & 0.19 \\
\hline & 120 & 3.32 & 1.03 & 0.31 \\
\hline & 150 & 3.32 & 1.16 & 0.35 \\
\hline & 180 & 3.25 & 1.92 & 0.59 \\
\hline & 210 & 3.25 & 2.01 & 0.62 \\
\hline \multirow[t]{7}{*}{$x$} & 0 & 3.89 & 1.01 & 0.26 \\
\hline & 15 & 3.89 & 0.79 & 0.20 \\
\hline & 60 & 3.89 & 0.62 & 0.16 \\
\hline & 90 & 4.23 & 1.39 & 0.33 \\
\hline & 150 & 4.23 & 1.73 & 0.41 \\
\hline & 180 & 4.23 & 1.73 & 0.41 \\
\hline & 230 & 4.23 & 2.87 & $0 . ; 8$ \\
\hline \multirow[t]{6}{*}{$\mathrm{XI}$} & 0 & 3.34 & 0.77 & 0.23 \\
\hline & 30 & 3.34 & 0.73 & 0.22 \\
\hline & 45 & 3.34 & 0.60 & 0.18 \\
\hline & 60 & 3.34 & 0.57 & 0.17 \\
\hline & 100 & 3.34 & 0.53 & 0.16 \\
\hline & 180 & 3.34 & 1.60 & 0.48 \\
\hline \multirow[t]{7}{*}{ XII } & 15 & 3.33 & 0.96 & 0.29 \\
\hline & 20 & 3.33 & 1.03 & 0.31 \\
\hline & 120 & 3.33 & 1.63 & 0.49 \\
\hline & 135 & 3.35 & 1.57 & 0.47 \\
\hline & 180 & 3.35 & 1.91 & 0.57 \\
\hline & 190 & 3.35 & 1.50 & 0.45 \\
\hline & 210 & 3.35 & 2.11 & 0.63 \\
\hline \multirow[t]{3}{*}{ XIII } & 190 & 3.60 & 2.30 & 0.64 \\
\hline & 200 & 3.60 & 2.99 & 0.83 \\
\hline & 215 & 3.60 & 2.92 & 0.81 \\
\hline \multirow[t]{7}{*}{ XIV } & 30 & 2.91 & 0.67 & 0.23 \\
\hline & 75 & 2.94 & 1.06 & 0.36 \\
\hline & 90 & 3.22 & 0.80 & 0.25 \\
\hline & 120 & 3.22 & 1.64 & 0.51 \\
\hline & 135 & 3.22 & 1.96 & 0.61 \\
\hline & 165 & 3.24 & 2.14 & 0.66 \\
\hline & 210 & 3.24 & 2.33 & 0.72 \\
\hline \multirow[t]{2}{*}{ Mean $\pm s D$} & $\bar{X}_{2}$ & $3.41 \pm 0.39$ & $z$ & $0.29 \pm 0.13$ \\
\hline & $\bar{X}_{\mathbf{8}}$ & $3.53 \pm 0.39$ & 4 & $0.62 \pm 0.10$ \\
\hline
\end{tabular}

\section{Animals with Rickets}

Four-week-old rats of the inbred type BD2 [43] were fed the McCollum rickets diet and carrots (Ca:P = 4:1) for 2-3 weeks. After this period of time the animals showed severe and extremely severe rickety changes. In every rat these changes were determined by a radiogram of the knee joint.
Results

\section{Normal Animals}

Figure 1 shows the effect of intravenous administration of vitamin $D_{3}$ on the TF/P-phosphate ratio in the proximal convolution of the rat kidney. In normal animals infused with $\mathrm{NaCl}(1.5 \mathrm{ml} / \mathrm{hr})$ the TF/P ranged from 0.53 to 0.84 , and the mean $\left(\bar{X}_{1}\right)$ was 0.67 \pm 0.09 (Table I). There was no apparent change in the $\mathrm{TF} / \mathrm{P}$ ratio after infusion of $\mathrm{NaCl}$ (Fig. 2). After infusion of vitamin $\mathrm{D}_{3}$ was started, and largely independent of the dosage $(1.800-20 \mathrm{U} / \mathrm{min} / 100 \mathrm{~g})$, intratubular concentration of phosphate decreased. This was followed by a decrease of the TF/P.phosphate (Fig. 1). The intratubular concentration of phosphate reached the lowest level from 60 to $120 \mathrm{~min}$ after the start of the infusion. The mean of all measured values $\left(\overline{\mathrm{X}}_{2}\right)$ was $0.35 \pm 0.11(P<0.001)$.

Table II shows that the glomerular filtration rate

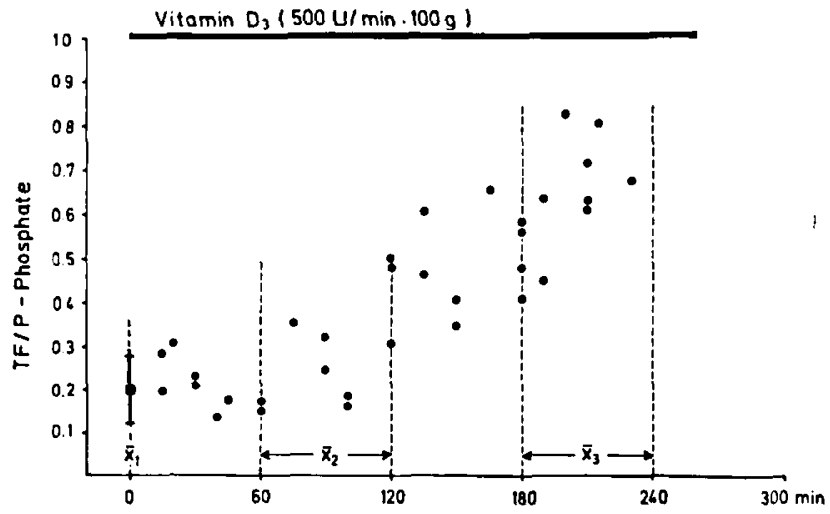

Fig. 3. The effect of infusion of vitamin $D$ on the TF/P-phosphate of parathyroidectomized rats. Vitamin $\mathrm{D}_{\mathbf{3}}$ dose, $500 \mathrm{U} / \mathrm{min} /$ $100 \mathrm{~g}$.

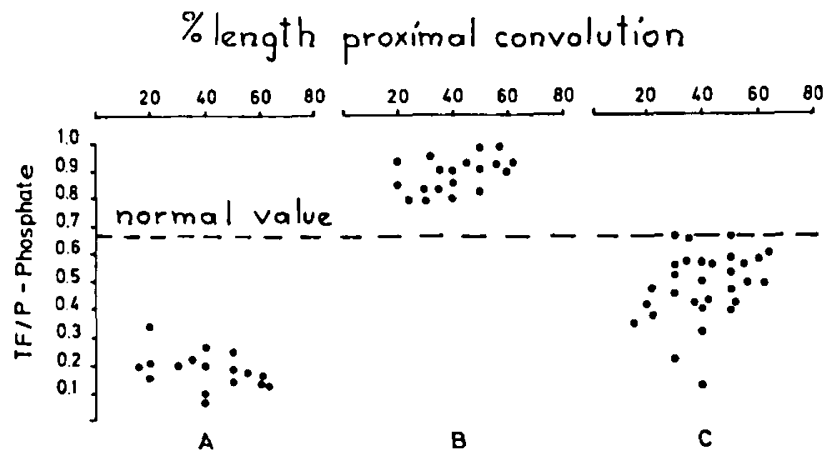

Fig. 4. Relation between TF/P-phosphate and tubular length. $A$ : Animals with rickets without phosphate load. $B$ : Animals with rickets with phosphate load. $C$ : Animals with rickets with phosphate load and vitamin $\mathrm{D}(500 \mathrm{U} / \mathrm{min} / 100 \mathrm{~g}$ body $\mathrm{wt})$. 
Table IV. Rachitic rats

\begin{tabular}{|c|c|c|c|c|c|c|}
\hline Rat & $\begin{array}{l}\text { Plasma phosphate } \\
\text { mmoles/liter }\end{array}$ & $\begin{array}{l}\text { Tubular } \\
\text { phosphate }\end{array}$ & TF/P-phosphate & $\begin{array}{c}\text { Tubule length, } \\
\%\end{array}$ & $\mathrm{GFR}, \mathrm{ml} / \mathrm{min} / \mathrm{kg}$ & $\begin{array}{c}\text { Serum } \\
\text { calcium, } \\
\mathrm{mg} / 100 \mathrm{ml}\end{array}$ \\
\hline \multirow[t]{3}{*}{ I } & 2.00 & 0.70 & 0.35 & 20 & 7.16 & 8.4 \\
\hline & 2.14 & 0.36 & 0.17 & 60 & 8.00 & \\
\hline & 2.16 & 0.43 & 0.20 & 18 & 7.78 & \\
\hline \multirow[t]{4}{*}{ II } & 2.21 & 0.31 & 0.14 & 58 & 5.42 & 4.8 \\
\hline & 2.16 & 0.30 & 0.14 & 45 & 11.40 & \\
\hline & & & & & 11.48 & \\
\hline & 2.21 & 0.35 & 0.16 & 21 & 9.92 & \\
\hline \multirow[t]{4}{*}{ III } & 1.70 & 0.36 & 0.21 & 21 & 11.30 & 2.7 \\
\hline & 1.80 & 0.14 & 0.08 & 40 & 11.62 & \\
\hline & & & & & 11.32 & \\
\hline & 1.70 & 0.17 & 0.10 & 39 & 11.28 & \\
\hline \multirow[t]{3}{*}{ IV } & 1.50 & 0.40 & 0.27 & 40 & 11.56 & 4.1 \\
\hline & 1.33 & 0.32 & 0.24 & 45 & 10.48 & \\
\hline & 1.61 & 0.32 & 0.20 & 25 & 11.40 & \\
\hline \multirow[t]{2}{*}{ V } & 1.61 & 0.35 & 0.22 & 28 & 12.72 & 7.2 \\
\hline & 1.80 & 0.31 & 0.17 & 64 & 10.42 & \\
\hline \multirow[t]{3}{*}{ VI } & 1.80 & 0.32 & 0.18 & 55 & 9.50 & 4.5 \\
\hline & 1.75 & 0.35 & 0.20 & 42 & 9.20 & \\
\hline & 1.75 & 0.33 & 0.19 & 45 & 5.96 & \\
\hline Mean $\pm s D$ & $1.84 \pm 0.25$ & & $0.19 \pm 0.06$ & & $9.89 \pm 2.09$ & \\
\hline
\end{tabular}

Table V. Phosphate-loaded rachitic rats

\begin{tabular}{|c|c|c|c|c|c|c|c|c|}
\hline Rat & $\underset{\substack{\boldsymbol{m} \\
\text { infusion }}}{,}$ & $\begin{array}{l}\text { Plasma phosphate } \\
\text { mmoles } / 1\end{array}$ & $\begin{array}{l}\text { Tubular } \\
\text { phosphate } \\
\text { ter }\end{array}$ & TF/P-phosphate & $\begin{array}{c}\text { Tubule } \\
\text { length, } \\
\%\end{array}$ & TF/P-inulin & $\mathrm{GFR}, \mathrm{ml} / \mathrm{min} / \mathrm{kg}$ & $\begin{array}{c}\text { Serum } \\
\text { calcium, } \\
\mathrm{mg} / 100 \mathrm{ml}\end{array}$ \\
\hline \multirow[t]{3}{*}{ I } & 30 & 6.70 & 5.36 & 0.80 & 40 & & & 6.8 \\
\hline & 70 & 6.55 & 6.16 & 0.94 & 20 & & & \\
\hline & 130 & 7.25 & 6.09 & 0.84 & 30 & & & \\
\hline \multirow[t]{3}{*}{ II } & 30 & 4.80 & 3.98 & 0.83 & 35 & & 10.94 & \\
\hline & 110 & 6.21 & 5.59 & 0.90 & 38 & & & \\
\hline & 200 & 7.20 & 6.70 & 0.93 & 44 & & & \\
\hline \multirow[t]{3}{*}{ III } & 95 & 5.00 & 4.50 & 0.90 & 60 & 1.50 & 11.98 & 8.4 \\
\hline & 180 & 7.90 & 6.71 & 0.85 & 20 & & & \\
\hline & 200 & 7.92 & 6.81 & 0.86 & 40 & & & \\
\hline \multirow[t]{4}{*}{ IV } & 90 & 3.42 & 3.08 & 0.90 & 33 & & 9.62 & \\
\hline & 105 & 5.43 & 5.37 & 0.99 & 50 & 0.98 & 9.88 & \\
\hline & 130 & 5.41 & 4.97 & 0.92 & 55 & 1.74 & 7.48 & \\
\hline & 180 & 7.00 & 6.51 & 0.93 & 62 & 2.16 & 8.58 & \\
\hline \multirow[t]{2}{*}{ V } & 75 & 4.28 & 3.38 & 0.79 & 28 & & & 9.0 \\
\hline & 145 & 6.52 & 6.19 & 0.95 & 32 & & & \\
\hline \multirow[t]{4}{*}{ VI } & 115 & 5.83 & 4.66 & 0.80 & 25 & & & \\
\hline & 160 & 6.56 & 5.64 & 0.86 & 40 & & & \\
\hline & 185 & 7.12 & 6.48 & 0.91 & 50 & 2.25 & 11.02 & \\
\hline & 215 & 7.34 & 7.34 & 1.00 & 57 & 1.58 & & \\
\hline Mean $\pm s D$ & & $6.25 \pm 1.38$ & & $0.90 \pm 0.10$ & & & $9.93 \pm 1.54$ & \\
\hline
\end{tabular}


(GFR), the level of phosphate in plasma, and TF/P-inulin ratio had not changed as compared with the control group (Table I). Subsequently, the concentration of phosphate in the lumen again increased, and after 180-240 min the TF/P, which had been $0.64 \pm 0.11$ $\left(\bar{X}_{3}\right)$, again was in the range of the starting values. At this time also the GFR, TF/P-inulin, and the phosphate concentration in plasma (Table II) were practically unchanged when compared with the control values. The tubular phosphate load was constant for all three periods $\left(\bar{X}_{1}=18.45, \bar{X}_{2}=18.83, \bar{X}_{3}=18.23\right.$ $\mu$ moles $/ \mathrm{min} / \mathrm{kg})$. In contrast to this, the calcium level during the second period $(9.47 \pm 0.34 \mathrm{mg} / 100 \mathrm{ml})$ showed a slight but significant decrease $(P<0.001)$ compared with the values exhibited by the control group $(10.16 \pm 0.36 \mathrm{mg} / 100 \mathrm{ml})$. During the third period, however, the calcium levels returned to control values $(9.80 \pm 0.91 \mathrm{mg} / 100 \mathrm{ml})$.

\section{Parathyroidectomized Animals}

The results of these studies are summarized in Table III. The TF/P-phosphate ratio of parathyroidectomized rats infused with $\mathrm{NaCl}(1.5 \mathrm{ml} / \mathrm{hr})$ ranged from 0.10 to 0.40 ; the mean $\left(\bar{X}_{1}\right)$ was $0.20 \pm 0.11$.

Table VI. Phosphate-loaded rachitic rats infused with vitamin D

\begin{tabular}{|c|c|c|c|c|c|c|}
\hline Rat & $\begin{array}{c}t, \\
\text { min after } \\
\text { infusion }\end{array}$ & $\begin{array}{r}\text { Plasma phosphate } \\
\text { mmo }\end{array}$ & $\begin{array}{c}\text { Tubular } \\
\text { phosphate }\end{array}$ & TF/P-phosphate & $\begin{array}{c}\text { Tubule length, } \\
\%\end{array}$ & $\begin{array}{c}\text { Serum } \\
\text { calcium, } \\
\mathrm{mg} / 100 \mathrm{ml}\end{array}$ \\
\hline \multirow[t]{3}{*}{$I$} & 70 & 5.00 & 2.12 & 0.42 & 22 & 5.8 \\
\hline & 110 & 5.14 & 1.80 & 0.35 & 15 & \\
\hline & 160 & 7.20 & 3.60 & 0.50 & 55 & \\
\hline \multirow[t]{3}{*}{ II } & 100 & 5.20 & 2.08 & 0.40 & 42 & \\
\hline & 185 & 7.15 & 3.36 & 0.47 & 50 & \\
\hline & 205 & 6.22 & 3.67 & 0.59 & 28 & \\
\hline \multirow[t]{5}{*}{ III } & 80 & 3.86 & 1.62 & 0.42 & 28 & 6.2 \\
\hline & 95 & 4.83 & 2.85 & 0.59 & 50 & \\
\hline & 135 & 5.28 & 2.64 & 0.50 & 64 & \\
\hline & 150 & 5.70 & 3.42 & 0.60 & 65 & \\
\hline & 185 & 6.02 & 4.09 & 0.68 & 50 & \\
\hline \multirow[t]{3}{*}{ IV } & 50 & 3.26 & 0.75 & 0.23 & 28 & 4.9 \\
\hline & 110 & 5.16 & 3.04 & 0.59 & 62 & \\
\hline & 155 & 5.90 & 3.19 & 0.54 & 48 & \\
\hline \multirow[t]{4}{*}{ V } & 60 & 4.21 & 0.55 & 0.13 & 44 & \\
\hline & 100 & 4.83 & 1.83 & 0.38 & 23 & \\
\hline & 115 & 5.02 & 2.41 & 0.48 & 22 & \\
\hline & 125 & 5.43 & 2.71 & 0.50 & 40 & \\
\hline \multirow[t]{4}{*}{ VI } & 80 & 5.00 & 2.30 & 0.46 & 28 & 6.0 \\
\hline & 95 & 5.06 & 2.17 & 0.43 & 42 & \\
\hline & 130 & 6.30 & 2.39 & 0.33 & 41 & \\
\hline & 160 & 6.84 & 3.90 & 0.57 & 43 & \\
\hline \multirow[t]{3}{*}{ VII } & 110 & 4.60 & 1.84 & 0.40 & 50 & \\
\hline & 150 & 5.40 & 3.08 & 0.57 & 41 & \\
\hline & 185 & 7.30 & 4.89 & 0.67 & 37 & \\
\hline \multirow[t]{5}{*}{ VIII } & 70 & 3.81 & 2.02 & 0.53 & 30 & 5.4 \\
\hline & 120 & 4.73 & 2.03 & 0.43 & 52 & \\
\hline & 135 & 5.01 & 2.80 & 0.56 & 55 & \\
\hline & 180 & 6.23 & 3.61 & 0.58 & 33 & \\
\hline & 195 & 6.69 & 4.41 & 0.66 & 35 & \\
\hline Mean $\pm s D$ & & $5.25 \pm 1.24$ & & $\begin{array}{r}0.48 \pm 0.18 \\
\bar{X}_{8} 0.61 \pm 0.02\end{array}$ & & \\
\hline
\end{tabular}


Compared with intact animals, the GFR, which was $5.03 \pm 0.49 \mathrm{ml} / \mathrm{min} / \mathrm{kg}$, was decreased $(P<0.001)$, and level of phosphate, $3.40 \pm 0.26 \mathrm{mmole} / \mathrm{liter}$ was increased $(P<0.001)$. Because of the low starting values in this group it was not possible to demonstrate a definite decrease of the TF/P-phosphate ratio $\left(\bar{X}_{2}=\right.$ $0.29 \pm 0.13)$ after the start of the vitamin $D$ infusion (Fig. 3). In some tests (Table III $B$, rat Nr.IX-XI) there was a slight but not significant decrease of the phosphate ratio. With continuous infusion of vitamin $D$, the $T F / P$-phosphate again showed a linear increase after $120 \mathrm{~min}$, after $180-240 \mathrm{~min}$ it had reached a mean $\left(\overline{\mathrm{X}}_{3}\right)$ of $0.62 \pm 0.10(P<0.00 \mathrm{l})$. At this time the phosphate level was $3.53 \pm 0.39$ mmoles/liter.

\section{Animals with Rickets}

As illustrated in Figure 4 and Table IV, the TF/P. phosphate ratio in rats with rickets $(A)$ ranged from 0.08 to 0.35 ; the mean was $0.19 \pm 0.06$. Compared with values of the control group, the GFR, $9.89 \pm 2.09$ $\mathrm{ml} / \mathrm{min} / \mathrm{kg}$, was significantly increased $(P<0.001)$. With a phosphate level of $1.84 \pm 0.25$ mmoles/liter a tubular load of $18.20 \mu$ moles $/ \mathrm{min} / \mathrm{kg}$ for animals with rickets could be calculated. To demonstrate the effect of vitamin $D$ in rats with rickets, the animals were loaded with phosphate $(0.15 \mathrm{mmole} / \mathrm{hr})$. Under this stress (Fig. 4B, Table V) (the mean level of phosphate was $6.25 \pm 1.38 \mathrm{mmoles} / \mathrm{liter}$ ) the $\mathrm{TF} / \mathrm{P}$-phosphate increased to values ranging from 0.79 to 1.00 (mean 0.90 \pm 0.10 ), and the GFR (Table V), which was $9.93 \pm$ $1.54 \mathrm{ml} / \mathrm{min} / \mathrm{kg}$, remained unchanged. When vitamin $\mathrm{D}$ was simultaneously added to the infusion of phosphate (Fig. $4 C$, Table VI), the ratio ranged from 0.13 to 0.67 (mean $0.48 \pm 0.18, P<0.001$ ). The large variation of this group was due to the micropunctures, which were carried out at different times during the vitamin $\mathrm{D}$ infusion.

Figure 5 shows that the TF/P-phosphate was lowest after the infusion had been running for $60 \mathrm{~min}$. Subsequently, in normal as well as in parathyroidectomized animals, the ratio again increased. After 180-240 min the $T F / P$ was within the range of the values of normal animals $\left(\bar{X}_{3}=0.61 \pm 0.02\right)$. Figure 5 also shows that the rats with rickets that had not received vitamin $D$ did not show the respective change under phosphate loading.

\section{Discussion}

The results show that in normal animals vitamin $\mathrm{D}$ increased the transtubular reabsorption of phosphate

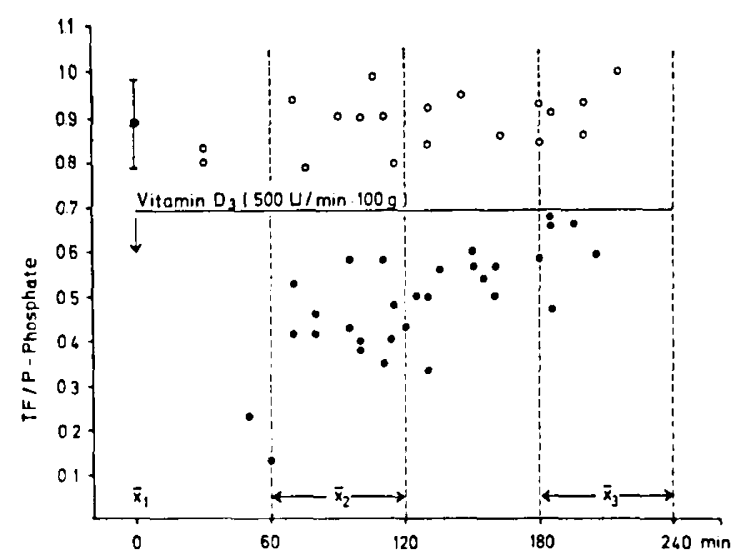

Fig. 5. The TF/P-phosphate ratios of phosphate-loaded animals with rickets without $(O)$ and with $(\bullet)$ vitamin $D$.

immediately after the start of the infusion. Since the GFR, the level of phosphate in plasma, and the TF/P. inulin ratio (the latter as a measure of the intratubular reabsorption of fluid) did not change, one can regard the increased reabsorption as a specific effect of vitamin D. This result was unexpected. In all the in vitro and in vivo studies of vitamin $\mathrm{D}$ action upon intestinal transport of calcium and phosphate, a latent period of 2 or 3 hr exists between the time of administration of vitamin $\mathrm{D}$ and demonstration of its physiological effect [14]. The reason for these varying results is not apparent at present. Experiments with the pure solvent without vitamin $\mathrm{D}$ had no effect on the TF/P-phosphate, and studies by Jesserer $[17,18]$ clearly showed that there is no different action between the vitamin $\mathrm{D}_{3}$ in watersoluble complex and other modifications. The only experiments in which addition of vitamin $\mathrm{D}$ directly to a metabolizing system has altered its function are those of Engstrom and DeLuca [7] in which kidney mito. chondrial preparations have been used.

This report shows clearly that vitamin $\mathrm{D}_{2}$ and $\mathrm{D}_{3}$ adcled in vitro dramatically stimulated the release of calcium. It may be that the filtrated vitamin $D_{3}$ in our experiments exerted an analogous effect on the Iuminal side of the tubular cells.

From the results presented in Table I it was possible to calculate the absolute amount of reabsorption of phosphate in the control group. The tubular reabsorption of phosphate at the end of the proximal convolution, with a TF/P-inulin of 2.5 and a mean TF/P. phosphate of 0.67 , was $13.51 \mu \mathrm{moles} / \mathrm{min} / \mathrm{kg}$ in normal animals. The tubular $\mathrm{PO}_{4}$ load of $18.45 \mu \mathrm{moles} / \mathrm{min} /$ $\mathrm{kg}$, corresponds to a fractional reabsorption of $73 \%$; i.e., in normal animals $73 \%$ of the filtrated phosphate is reabsorbed after passage through the first two-thirds 
of the proximal tubule. This reabsorption corresponds to the values which have been reported by Strickler $e t$ al. [31]. He found, also in micropuncture studies, a phosphate reabsorption of $10.10 \mu \mathrm{moles} / \mathrm{min} / \mathrm{kg}$ and a fractional reabsorption of $76 \%$.

If the reabsorption of phosphate under the influence of vitamin $D$ is calculated for the time $\bar{X}_{2}$, when $T F /$ $P$-inulin end-proximal ratio is 2.5 , one finds a reabsorption of $15.28 \mu \mathrm{moles} / \mathrm{min} / \mathrm{kg}$, or a fractional reabsorption of $86 \%$. Thus, tubular transport of phosphate under the influence of vitamin $D$ showed a definite increase in normal rats. Figure 1 and Table II also show that a continuous infusion of vitamin $D$ for $180-240$ $\min \left(\bar{X}_{3}\right)$ increased the TF/P-phosphate to $0.64 \pm 0.11$. Since the concentration of the phosphate in plasma had not changed, the increase must have been due to an increased concentration of the intratubular phosphate. This reactive inhibition of the phosphate transport with a consequent rise of the TF/P-phosphate, however, was only effective until the normal TF/P ratio had been once again reached. This means that only the reabsorption which was under the effect of vitamin D was inhibited; further inhibition of phosphate transport did not materialize. This finding corresponds to the observations of Watchorn [33] as well as of Winberg and Zetterström [34], who reported that increased phosphate excretion in the urine does not occur even with vitamin $\mathrm{D}$ intoxication. The GFR, $7.16 \pm 1.27 \mathrm{ml} / \mathrm{min} / \mathrm{kg}$, is not increased compared with findings at times $\bar{X}_{1}$ and $\bar{X}_{2}$. For time $\bar{X}_{3}$ a tubular load of $18.23 \mu$ moles $/ \mathrm{min} / \mathrm{kg}$-with a TF $/ \mathrm{P}$ inulin of 2.5 and an absolute reabsorption of phosphate of $13.95 \mu \mathrm{moles} / \mathrm{min} / \mathrm{kg}$ can be calculated. This amount corresponds to a fractional reabsorption of $74 \%$.

As has been shown by the experiments of Kodicek [20], the property of vitamin D of increasing transport of phosphate in the proximal tubule closely correlates with its distribution in the nephron. During autoradiographic examinations Kodicek found, in the first one-third of the proximal convolution, a strong accumulation of ${ }^{14} \mathrm{C}$-labeled vitamin $\mathrm{D}$ but no or only minimal activity in the other parts of the nephron. We know from numerous studies, however, that transport of phosphate takes place in the proximal tubule. It remains undetermined where the tubular phosphate secretion observed during the terminal stage of renal failure is located [8]. The studies of Nicholson and Shepherd [24] indicate that distal secretion of phosphate, which has been proved to exist in lower animal species, actually also takes place in mammals.
It is still not clear in what manner vitamin $\mathrm{D}$ affects reabsorption of phosphate. Since it was shown by micropuncture studies that parathyroid hormone inhibits tubular reabsorption of phosphate in the proximal convolution $[4,10]$, this effect could either result from inhibition of the secretory activity of the parathyroid glands or be a direct effect of vitamin $D$ on cellular phosphate transport. Our studies were also unable to solve this problem. The findings suggested, however, that this is not just a problem of dealing with inhibition of parathyroid function, but also one involving a direct effect on the tubular transport system.

In parathyroidectomized animals, the studies showed a decreased $T F / P$-inulin of 2.2 at the proximal end. With a mean TF/P-phosphate of $0.20 \pm 0.08$ for time $\bar{X}_{1}$ an absolute reabsorption of $15.55 \mu$ moles/ $\mathrm{min} / \mathrm{kg}$ or a fractional reabsorption of $91 \%$ can be calculated. Even though tubular transport of phosphate with a constant GFR is limited by a transport maximum [25], it was found that in some tests with vitamin $D$ the reabsorption of phosphate also increased in parathyroidectomized animals. Analogous studies on the small intestine of the rat also indicated a direct effect of vitamin $\mathrm{D}$ on phosphate reabsorption and these results could be extrapolated to the renal tubule. These tests showed that vitamin $\mathbf{D}$ increases the reabsorption of phosphate against a concentration gradient [14]. Furthermore, since the reabsorption-promoting action occurred immediately after the start of the infusion, and since it again decreased after 120 min, we presume that an action on the secretory activity of the parathyroid gland was unlikely. In contrast it was possible that vitamin $D$ inhibited the activity of parathyroid hormone on the cell, e.g., by inhibiting the formation of cyclic $3^{\prime}, 5^{\prime}$-adenosine monophosphate (AMP). The observations of Russell et al. [27] that cyclic AMP not only plays a role in the action mechanism of the anticliuretic hormone, but also plays a role in the effect of parathyroid hormone on the kidney, supports this hypothesis. It will only be possible to prove this, however, when we know more about the biochemical mode of action of these two substances.

It remains undetermined why the initially definite reabsorption increase once again decreased in all test groups after 90-120 min, or why the TF/P-phosphate showed a linear increase. Albright and Reifenstein [2] have already observed that vitamin $D$ in high doses $(200,000-400,000 \mathrm{U} / 24 \mathrm{hr})$ increased phosphate excretion in the urine of patients with hypoparathyroidism. Similar findings were obtained by clearance studies in parathyroidectomized rats [5]. Hitherto, attempts to 
contrast this reversal of the effect as a so-called pharmacological effect of high doses to the known physiological effect have not been convincing [15].

Our studies also failed to show a relation to the dose of vitamin $\mathrm{D}$. The observation that the calcium level does not increase during infusion of vitamin $D$, whereas the symptoms of vitamin $\mathrm{D}$ intoxication depend significantly on the magnitude of the calcium concentration, also argue against the existence of vitamin D intoxication [30, 34]. Furthermore, a decrease of the GFR, described in dogs after the administration of high, toxic doses of vitamin D $[13,14,21]$, was not observed. Thus, one would have to discuss the exhaustibility of a metabolite of vitamin $D$ in the sense of DeLuca $e t$ al. [6], a reactively increased breakdown of the vitamin (e.g., in the liver) or developing resistance to the physiological activity of vitamin D, similar to the pathological appearance of vitamin D-resistant rickets. High doses of vitamin D, however, may also produce an increase in the secretion of thyrocalcitonin. On the basis of the reabsorption-inhibiting effect of thyrocalcitonin [11], it would be possible to explain the increase in the TF/P.phosphate. It is certain, however, that the observed "inactivation" was not an effect of parathyroid hormone since it was also seen in parathyroidectomized animals and it was not the result of toxic renal damage because renal function was controlled during the entire experiment.

As shown by tests on animals with rickets, the TF/ P-phosphate mean was $0.19 \pm 0.06$. Initially, this observation was surprising since it led to the assumption that reabsorption of phosphate was almost complete in animals with rickets. Assuming a TF/P-inulin at the proximal end of 2.5 , one can calculate the absolute reabsorption and one finds a reabsorption rate of 16.82 $\mu$ moles $/ \mathrm{min} / \mathrm{kg}$ and a fractional reabsorption of $92 \%$. This high reabsorption and the low TF/P ratio, however, was a result of the low content of phosphate in the feed. The ratio of reabsorbed phosphate to filtrated phosphate approaches a value 1 when administrated phosphate approximates 0 [16]. With the McCollum diet, the $\mathrm{Ca} / \mathrm{P}$ ratio was $4: 1$. To keep the concentration of phosphate in the blood at approximately normal levels, the filtrated phosphate is almost completely reabsorbed in the proximal tubule as a compensatory mechanism. As a result of this, the TF/ $P$-phosphate ratio showed a strong decrease with an increasing tubular length, or in the final urine collection the excretion of phosphate was exceedingly small. Under phosphate loading this was different. In this situation, with a mean phosphate level of $6.25 \pm 1.38$ mmoles/liter, an increase of the TF/P value to $0.90 \pm$ 0.10 took place $(P<0.001)$. With a TF $/ P$-inulin of 2.2 at the proximal end we calculated a fractional reabsorption of only $59 \%$ in this group. If vitamin $\mathrm{D}$ is simultaneously infused with the phosphate load, however, one finds, when examining only the $T F / P$ ratios up to $120 \mathrm{~min}$ after the start of the infusion, a TF/P. phosphate ratio of $0.42 \pm 0.15(P<0.001)$. Assuming that the GFR and the TF/P-inulin ratios were not changed by vitamin $D$, as seen in normal animals, we found a fractional reabsorption of $78 \%$. Thus, with vitamin $D$ infusions it was possible to increase phosphate reabsorption by $32 \%$.

\section{References and Notes}

1. Albright, F.; Bloomberg, E., Drake, T. G., and Sulkowitch, H. W.: A comparison of the effects of AT 10 (dihydrotachysterol) and vitamin $D$ on calcium and phosphorus metabolism in hypoparathyroidism. J. Clin. Invest., 17: 317 (1938).

2. Albrigit, F., and Reifenstein, E. C.: The Parathyroid Glands and Metabolic Bone Disease. (Williams \& Wilkins, Baltimore, 1948).

3. AU, W. Y. W., AND Raisz, L. G.: Effect of vitamin D and dictary calcium on parathyroid activity. Amer. J. Physiol., 209: 637 (1955).

4. CArone, F. A.: Micropuncture study of renal phosphate excretion and action of parathyroid hormone. Clin. Res., 12: 249 (1964).

5. Crawford, J. D., Gribetz, D., and Talbot, N. B.: Mechanism of renal tubular phosphate reabsorption and the influence thercon of vitamin $\mathrm{D}$ in completely parathyroidectomized rats. Amer. J. Physiol., 180: 156 (1955).

6. Deluca, H. F., Lund, J., Rosenbloom, A., and Lobeck, C. C.: Metabolism of tritiated vitamin $D_{a}$ in familial vitamin $D$. resistant rickets with hypophosphatemia. J. Pediat., 70: 828 (1967).

7. Engstrom, G. W., and DeLuca, H. F.: The action of vitamin $\mathrm{D}$ in vivo and in vitro on the release of calcium from kidney mitochondria. J. Biol. Chem., 237: PC974 (1962).

8. Fischer, V., Heidland, A., Klütsch, K., and Gekle, D.: Phosphat- und Harnsäuretransport bei terminaler Niercninsuffizienz. Verh. Deut. Ges. Inn. Med., 74: 1064 (1968).

9. Garnett, E. S., Parsons, V., and Veall, N.: Measurement of glomerular filtration-rate in man using a ${ }^{51} \mathrm{Cr} /$ edetic acid complex. Lancet, 1: 818 (1967).

10. Gekle, D., and Kossmann, K.: Der Einfluß von Thyreocalcitonin auf die Phosphatreabsorption der Niere (Mikropunk. tionsuntersuchungen). Mschr. Kinderheilk., 116: 308 (1968).

11. GEKLE, D., AND KURTH, G.: Mikropunktionsuntersuchungen über den Einfluß von Thyrcocalcitonin auf die renale Phos. phatreabsorption parathyreoidektomierter Ratten. VI. Symp. Ges. Nephrol., Wien, 1968. Wiener Med. Akad., Wien, 1969.

12. HaM, A. W., Littiner, N., Drake, T. G. H., Robertson, E. C., ANd Tisdall, F. F.: Physiological hypertrophy of the parathyroids, its cause and its relation to rickets. Amer. J. Pathol., 16: 277 (1940).

13. Harrison, H. E., and Harrison, H. C.: The renal excretion 
of inorganic phosphate in relation to the action of vitamin D and parathyroid hormone. J. Clin. Invest., 20: 47 (1941).

14. Harrison, H. E., AND Harrison, H. C.: Theories of vitamin D action. In: Transfer of Calcium and Strontium across Biological Membranes, p. 299 (Academic Press, New York, 1963).

15. Hövels, O., AND Reiss, D.: Physiologie und Stoffwechsel des D.Vitamins. Ergeb. Inn. Med. Kinderheilk. N.F., 11: 206 (1959).

16. Hungerland, H.: Calcium- und Phosphatstoffwechsel. In: S. J. Thannhauser: Lehrbuch des Stoffwechsels und der Stoffwechselkrankheiten, p. 865 (Neu bearbeitet von F. Bertram. Thieme, Stuttgart, 1957).

17. JESSERER, H.: Über die Wirkungsunterschiede verschiedener Lösungen von Vitamin $D_{2}$ und $D_{3}$ bei oraler, intravenöser und intramuskulärer Zufuhr. Wien. Klin. Wochenschr., 67: 49 (1955).

18. JESSERER, H.: Vergleichende Untersuchungen der Blutkalksteigernden Wirkung von Vitamin $D_{2}, D_{3}$, Dihydrotachysterin $_{2}$ und Dihydrotachysterin ${ }_{3}$ beim Menschen. Klin. Woch. enschr., 36: 263 (1958).

19. KLEIN, R., AND Gow, R. G.: Interaction of parathyroid hormone and vitamin $D$ on the renal excretion of phosphate. J. Clin. Endocrinol. Metab. 13: 271 (1953).

20. KoDicEK, E.: Turnover and distribution of vitamin D and its mode of action. In: Transfer of Calcium and Strontium across Biological Mcmbranes, p. 185 (Academic Press, New York, 1963).

21. Loudon, M., AND ZWEYMÜLLER, E.: Beitrag zur experimentellen Vitamin D.Vergiftung beim Hund. III. Mitt. Z. Exp. Med., 129: 325 (1957).

22. LOWRY, O. H., ROBERTS, N. R., LEINER, K. Y., WU, M. L., AND FARR, A. L.: The quantitative histochemistry of brains. I. Chemical methods. J. Biol. Chem., 20: 71 (1954).

23. Ney, R. L., Kelly, G., ANd Bartter, F. C.: Actions of vitamin $D$ independent of the parathyroid glands. Endocrinology, 82: 760 (1968).

24. Nicholson, T. F., AND Shepherd, G. W.: The effect of dam. age to various parts of the renal tubule on the excretion of phosphate by the dog's kidney. Can. J. Biochem., 37: 103 (1959).

25. Pirts, R. F.: Physiology of the Kidney and Body Fluids. (Year Book Medical, Chicago, 1964).

26. Rupp, W., AND SwOBOdA, W.: Untersuchungen des PO.Stoffwechsels bei Vitamin D-resistenter Rachitis (Phosphatdiabetes). III. Mitt.: Ergebnisse bei Uber- und Unterfunktion der Nebenschilddrüsen. Helv. Paediat. Acta, 11: 256 (1956).
27. Russel, R. G. G., Casey, P. A., AND Fleisch, H.: Stimulation of phosphate excretion by the renal arterial infusion of 3'5'-AMP (cyclic AMIP)-A possible mechanism of action of parathyroid hormone. Calcified Tissue Res., 2: 54 Suppl. (1968).

28. Stalder, G.: Zur renalen Wirkung des Vitamin $D_{2}$. Ann. Pacdiat., 186: 90 (1956).

29. Stalder, G.: Endogene Phosphat-Clearance bei Vitamin-D Mangelrachitis und rachitogener Tetanie: Ihre Beeinflußung durch Vitamin $D_{2}$. Int. Z. Vitaminforsch., 27: 382 (1957).

30. Stapleton, T., AND Evans, E. W.: Idiopathie hypercalcaemia in infancy. Helv. Pacdiat. Acta, 10: 149 (1955).

31. Strickler, J. C., Thompson, D. D., Klose, R. M., ANd GiebISCH, G.: Micropuncture study of inorganic phosphate excretion in the rat. J. Clin. Invest., 43: 1596 (1964).

32. Ullkici, K.-J., ANd Hampel, A.: Eine einfache Mikrocuvette für Monochromator Zeiss und Beckman Modell DU. Pflügers Arch. Ges. Physiol., 268: 177 (1958).

33. WATchorn, E.: Absorption and excretion of calcium and phosphorus by rats receiving excessive doses of irradiated ergosterol. Biochem. J., 24: 631 (1930).

34. Winberg, J., AND Zetterström, R.: Cortisone treatment in vitamin D intoxication. Acta Paediat. Scand., 45: 96 (1956).

35. ZWeymülter, E.: Die Wirkung von Vitamin D auf die Niere, unter besonderer Berücksichtigung der Nierenfunktion bei der D.Hypervitaminose. 1, und 2. Teil Neue Öster. Z. Kinderheilk., 2: 116, 178 (1957).

36. Obtained from Zentralinstitut für Versuchstierzucht, Hannover, Germany.

37. Althromin

38. Leitz-Monla, E. Leitz, Gmbtt, Wetzlar, West Germany.

39. Vi-De 3 Hydrosol Wander.

40. ${ }^{14} \mathrm{C}-$ Inulin and ${ }^{51} \mathrm{Cr}$-EDTA, Farbwerke Hocchst AG, Frankfurt, Germany.

41. Packard Instrument Company, Downers Grove, Ill.

42. Beckman Instruments, Inc., Fullerton, Calif.

43. Rats obtained from Forschungsinstitut für Milchvitaminierung, Frankfurt, Germany.

44. Supported by the Deutsche Forschungsgemeinschaft, Grant Ge 154/4, Bad Godesberg, Germany.

45. Presented in part at the IVth International Congress of Nephrology, Stockholm, Swcden, June, 1969.

46. Requests for reprints should be addressed to: Dr. D. Gekle, Universitäts-Kinderklinik, 8700 Würzburg, Germany.

47. Accepted for publication March 9, 1970. 